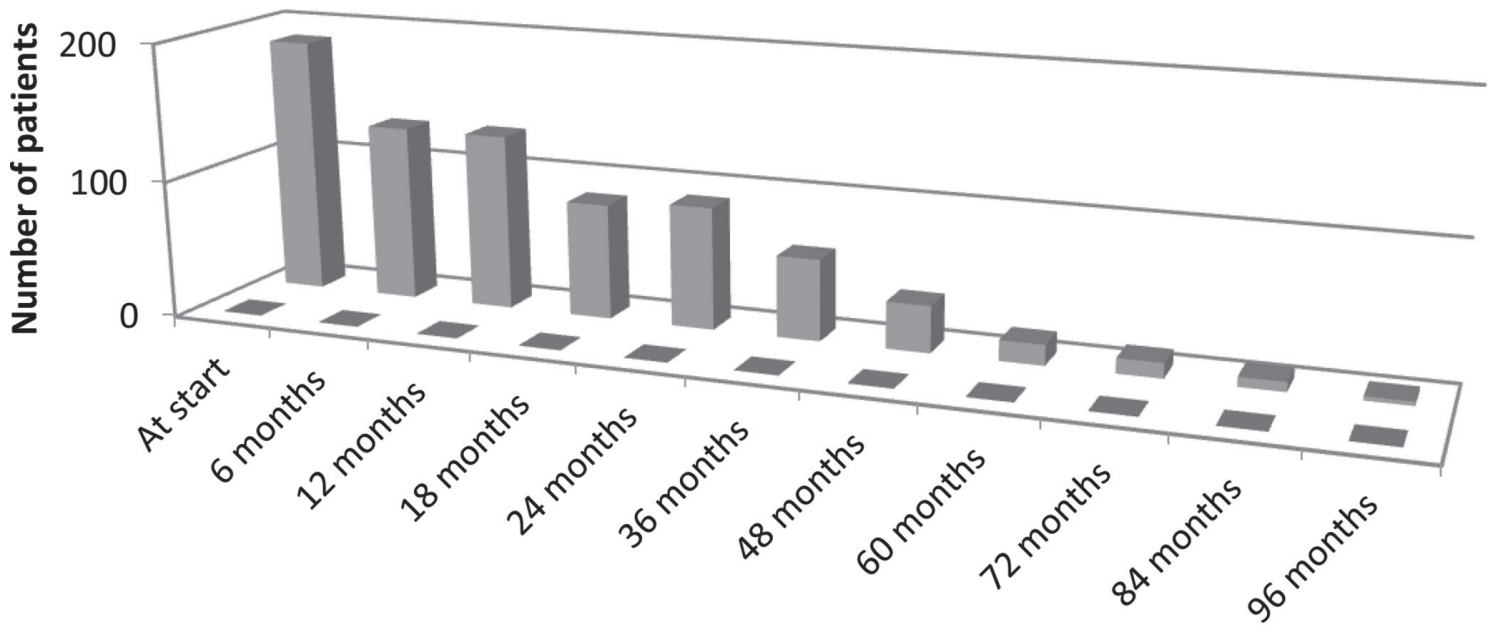

Time since started on Macrolide Therapy

NTM in sputum Total number of patients with sputum analysed

Abstract S39 Figure 1 NTM infection in sputum of patients on long term macrolides

randomised controlled trials involving larger populations of patients are required to confirm the benefits and harms.

\section{S40 A RETROSPECTIVE STUDY INTO THE CLINICAL RELEVANCE OF ISOLATING NON-TUBERCULOUS MYCOBACTERIA IN PULMONARY SAMPLES}

${ }^{1} \mathrm{H}$ Burgess, ${ }^{2} \mathrm{~S}$ Cowman, ${ }^{2} \mathrm{~A}$ Jones, ${ }^{2} \mathrm{R}$ Wilson, ${ }^{2} \mathrm{MR}$ Loebinger. ${ }^{1}$ St George's, University of London, London, UK; ${ }^{2}$ Royal Brompton Hospital, London, UK

\subsection{6/thoraxjnl-2016-209333.46}

Introduction and objectives There are in excess of 160 species of non-tuberculous mycobacteria (NTM), the minority of which can cause infection predominantly in patients with an underlying respiratory disease such as bronchiectasis or cystic fibrosis. Isolation of NTM in the respiratory tract does not always signify infection and can represent a transient infection, colonisation or active infection. The American Thoracic Society (ATS) criteria for the diagnosis of NTM infection can be used to help assess clinical relevance and the need to treat patients. A retrospective study was conducted to assess the clinical relevance of different species of NTM isolation.

Methods A database of microbiology results at a specialist trust was reviewed for patients with positive NTM isolates between January 2005 and December 2010. These patients were assessed against the ATS diagnostic criteria of NTM lung disease. Patient records for those who met the microbiological criteria records were reviewed for demographics, underlying condition and course of infection. Patients were followed up for a minimum of 5 years, or until discharge/death.

Results Five hundred and fifty-five (555) patients with positive NTM cultures were found, 281 (51\%) of whom met the ATS microbiological criteria. $70(13 \%)$ patients met the radiographic criteria and so were likely to have an active infection. This varied by species: M. Avium Complex (MAC) (21\%), M. malmoense (20\%), M. abscessus (18\%) and M. kansasii (17\%) were most likely to cause changes on CT, and species such as M. fortuitum (1.6\%) and M. gordonae (0\%) were less likely. The proportion of patients treated also varied by species, with $M$. abscessus (45\%), M. kansasii (23\%) and MAC (20\%) most likely to be treated. Five-year mortality for all patients who met the microbiological criteria was $21.4 \%$ and was significantly associated with meeting the full ATS criteria, as shown by Figure 1. Five-year survival and the patient's underlying condition also varied by species.

Conclusions Clinical relevance of NTM isolation varies by species, clinical symptoms and underlying condition. The decision to treat is influenced by these factors in addition to the ATS criteria.

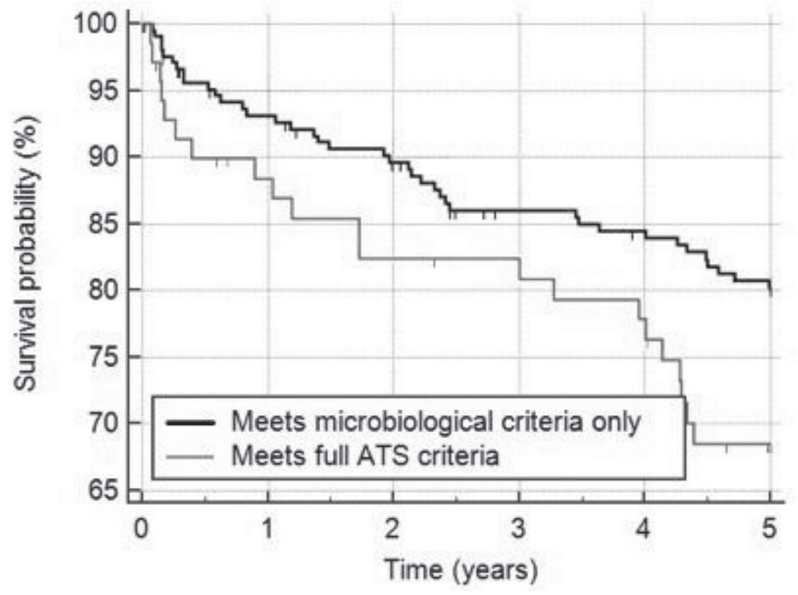

Abstract S40 Figure 15 -year survival of patients who meet full ATS criteria versus thode who only meet microbiological criteria $N=281$ ( $p$ $=0.041$ ) 